\title{
Seeing emotions without mindreading them
}

\section{Joulia Smortchkova}

\section{Introduction}

Can we see happiness in our friend's face? According to the direct perception approach the answer is positive: we can perceive emotions and by perceiving them we understand and ascribe them to others (Gallagher, 2014; Krueger \& Overgaard, forthcoming; Zahavi, 2011). In direct perception, emotions as mental states are not "hidden" from the senses and are immediately accessible to the perceiver. For example, Zahavi writes that in direct perception "my understanding of the other's psychological state can be said to be direct in the sense that that state is my primary intentional object." (2011, p. 548). Krueger pursues a similar idea when he writes: "According to Scheler, we can in fact see the mental states of others within the dynamics of their expressive behavior. There is no need to posit an additional extra- perceptual cognitive mechanism" (Krueger, forthcoming, p. 6).

On the opposite side of the spectrum, theorists of mindreading working in the simulation theory and theory theory frameworks give a negative answer: there is a non-perceptual multi- step process (involving either simulation or inference) that starts from the perception of low- level features of the facial display and ends up with emotion recognition (Goldman, 2006; Jacob, 2011).

For example, Jacob writes: "The facial expression of another's disgust might be difficult to discriminate from the facial expression of other affective states. [...] Thus, only by representing relevant contextual cues might one be able to disambiguate between the facial expression of another's experience of respectively pain and disgust. If so, then one cannot perceive the other's disgust in the other's facial expression alone, independently of the perception of contextual cues which are not part of the other's expression." (Jacob, 2011, p. 532).

In assessing who is right in this debate, it is useful to disentangle two different issues. The first issue is whether emotions can be perceptually experienced. This is distinct from the question of whether emotions can be immediately ascribed to others on the basis of perception. Intuitively one could say that if emotions can be perceptually experienced, they also can be immediately ascribed to others, on the model of epistemology of perception where experience is prima facie justification for ascriptions of properties to objects. This claim, however, only works if we focus on epistemology of emotion ascription and not on the psychological mechanisms of emotion ascription on the basis of perception. If we want to build up a perception-based model of the mechanisms of emotion ascription more than the appeal to this assumption has to be shown. The aim of this paper is to provide the first steps for the construction of a perception-based model of emotion ascription. 
When arguing in favor of emotion perception, the direct perception approach sometimes runs together several conceptually distinct claims. Yet, when inquiring about perception and attribution of emotions, we should try to distinguish at least four different issues:

1. The ontological question. What are emotions? What is the best theory of emotions?

2. The perceptual question. Can emotions be a target of perception?

3. The conceptualization question. How do we categorize and conceptualize emotions?

4. The mindreading question. Which mechanisms do we employ when we attribute emotions as mental states to others? What is the best theory of mindreading for emotions and mental states in general?

The direct perception case for emotion perception can be reconstructed as a chain of the following sub-theses:

1. Emotions as mental states are expressed/embodied in behavior, so to perceive the behavioral manifestations of emotions is to perceive the emotions as such (answer to question 1)

2. One can experience in perception properties and objects that go beyond traditionally perceptual features, such as colors, shapes, orientations, etc. (answer to question 2)

3. Emotions as mental states are already pre-categorized in perception (answer to question 3)1. Given that emotions as mental states are embodied, categorizing an emotion is already categorizing it as a mental state.

4. And finally, attribution is: a) perception-based and b) stems from interaction among social subjects rather than from "cold" cognitive mechanisms (answer to question 4).

A thorough discussion of these four theses is beyond the scope of this paper, even if I will touch upon the relation between question 2 and questions 3 and 4 . I think that the lack of clarity in distinguishing these four issues often undermines the direct perception approach (see Jacob 2011). If one could prove the truth of each sub-theses, then the case for direct perception would be stronger as it would be closely connected with the mechanisms underlying the claims.

My aim in this paper is to assess the first facet of direct perception theories: their appeal to perception in emotion recognition. I defend the claim that we can see emotions and not only infer them on the basis of low-level perceptual cues. However, I take this claim cum grano salis and I defend it separately from the other three sub-theses above. I distinguish between perceiving emotions and perceiving emotions as mental states. In order to show that emotions are perceived as 
mental states, one would need to take a very strong stance on the first question, endorse some version of the James-Lange theory and argue that in seeing the behavioral manifestations of emotions in others we do not merely see behaviors, but we see behaviors as integral components of the emotional state (Gallagher, 2004; Zahavi, 2011). Moreover, to perceive an emotion as a mental state is to be able to categorize and recognize it as such, to see it epistemically (see Section 2). Take as an example the case of visual agnosia: a patient with visual agnosia can see something without being able to categorize what she sees under such and such category. Visual agnosia has been used to study different stages in visual processing from detection of sensory features to full-blown recognition (Farah, 2004). Such a stage-based picture can be extended to emotion perception. Note, however, that saying that emotion perception might come in stages does not imply endorsing the inferentialist approach: a subject is not at all aware of these stages and the perceptual processes work beyond the subject's awareness.

Emotions are intuitively rather high-level phenomena. They are high-level in opposition to those properties that seem to be more obviously perceptual, such as sensory properties, for example shades, shapes, colors, etc. This casts doubt on the hypothesis that they can be truly perceived rather than inferred or cognized. But this hypothesis is not as absurd as it prima facie seems. In philosophy of perception there is an ongoing debate about the reach of perceptual experience: can we experience more than just shapes, sizes, colors, pitches, etc.? This debate is often called the debate on rich or liberal perceptual content (Hawley \& Macpherson, 2011). Since the question of emotion perception also concerns the ability of perceptual experience to include high-level properties I analyze the potentially fruitful hypothesis of emotion perception as a subset of the debate on the liberal content view.

The rest of the paper will be structured as follows. In section 2, I will frame the debate on emotion perception within Dretske's distinction between simple and epistemic seeing. In section 3 I answer a challenge to emotion perception. The challenge appeals to the presence of contextual influence to show that emotions cannot be perceived, but are inferred. In section 4 arguments and evidence in favor of emotions as targets of perception are introduced. In section 5 the discussion of neural substrates of emotion perception leads to a two-fold distinction: between perception and resonance on the one hand, and between emotion perception and emotion mindreading on the other hand.

\section{Kinds of seeing}

The first issue is what seeing means in discussing emotion perception. To distinguish uses of seeing one can appeal to the distinction between simple and epistemic seeing as tools for evaluating two options for direct perception (Dretske, 1969; Spaulding, in preparation). Simple seeing is a state 
that satisfies the following conditions:

1. [negative condition] the subject of the state needs no concepts of ' $a$ ' or beliefs about ' $a$ ' in order to simply see 'a' (Dretske, 1969, p. 4);

2. [positive condition] the subject simply sees ' $a$ ': ' $a$ ' is visually differentiated from its immediate environment by the subject (Dretske, 1969, p. 20).

Epistemic seeing (also called "seeing that"), on the other hand, is a belief state that is both perceptual and cognitive, which requires concept possession for the subject to enjoy the state and is factive (as in "seeing that this is a red apple"). It is a perceptual belief based on how things look. Such seeing allows us to represent the world as being a certain way. For example, to see that the cup is on the table, I must possess the concepts CUP and TABLE. There are two varieties of epistemic seeing.

A subject sees that $\mathrm{b}$ is $\mathrm{P}$ in a primary epistemic way only if:

1. $\mathrm{b}$ is $\mathrm{P}$.

2. The subject simply sees $b$.

3. The conditions under which the subject simply sees $b$ are such that $b$ would not look the way it now looks to the subject unless b were P (Dretske, 1969, pp. 78-79).

A subject sees that $\mathrm{b}$ is $\mathrm{P}$ in a secondary epistemic way only if:

1. $\mathrm{b}$ is $\mathrm{P}$.

2. The subject sees $\mathrm{c}(\mathrm{c}=b)$ and sees (primarily) that $\mathrm{c}$ is $\mathrm{S} .5$

3. Conditions are such that c would not be S unless b were P (Dretske, 1969, p. 154).

In some cases, direct perception claims seem to share some features with epistemic perception (for example in the idea of "smart perception" (Gallagher, 2008). Spaulding (in preparation) proposes an interpretation of direct perception in terms of epistemic seeing. In Dretske's view seeing emotions is a case of secondary epistemic seeing. We know that someone is angry by observing the external signs of her anger, in the same way as we know that the level of the fuel in the tank is low by looking at the gauge (Dretske, 1973). Contrary to Dretske, Spaulding proposes something more akin to primary epistemic seeing, in which seeing that someone is angry is a basic perceptual belief. However, if direct perception is an instance of epistemic seeing, it is not incompatible with extraperceptual approaches to emotion recognition, since there are cognitive processes involved in the formation of the perceptual belief, which might be based on the simple seeing of only low-level 
features.

But then the direct perception proposal becomes less interesting. Other proponents of direct perception lean toward the simple seeing interpretation, for example, Zahavi, when he talks of the emotion as being one's primary intentional object or Krueger, when he emphasizes the absence of extra-perceptual mechanisms. I suggest to keep the interpretation of direct perception in terms of simple seeing and explore the possibility of whether emotions can be simply seen before the ability to form perceptual beliefs and before recognition. Sections 4 and 5 present positive evidence in favor of this claim.

The second issue is whether we perceive emotions just like we perceive objects and their properties. In some cases direct perception of emotions is presented as a sui generis perception, especially dedicated mental states ("In intersubjective contexts, visual perception of the face of the other is not equivalent to glancing at an object", Gallagher, 2014, p. 3). In other cases it is presented as a case of object perception that extends to mental states ("But — and this is an old insight found for instance in the work of many Gestalt psychologists - whenever we perceive an object, we perceive it in a perceptual field." Zahavi, 2011, p. 547). I think that a fruitful discussion should rather be based on the hypothesis that emotion perception works similarly in many respects to object perception. First of all, we have extensive knowledge of the cognitive mechanisms involved in object perception and we could use this knowledge to assess the hypothesis of emotion perception. In order to claim that emotion perception is truly perceptual one would have to show that it shares many properties with the cases of perception we already know, for instance object perception. Second, perceiving objects can have two readings: a restricted reading, only as perception of material solid fixed objects, or as perception of the natural objects in our surroundings, which can include events (such as launching, Michotte, 1954), natural scenes (such as a gist of a landscape) and so on. If we start from the second reading the proposal that emotions perception works as object perception becomes less reductionist. Third, this does not force us to endorse the idea that emotions are perceived just as objects. There is evidence that even objects are not treated uniformly by the visual system (Jacob \& Jeannerod, 2003; Milner \& Goodale, 1995): sometimes perception feeds objects into the conceptual- recognitional faculties, sometimes it feeds objects into action mechanisms. Emotions may be perceived similarly to objects, yet remain somehow special. For example, emotions may be specially linked to empathy in a way in which tables and chairs are not, may elicit some actions (being drawn toward a smiling face and being repulsed by a frowning face), and the neural substrata for emotion perception might be different from the neural substrata of object perception.

The third and last issue is the definition of "direct". The notion of direct perception is not easy to 
pin down, since the same term has been used for a variety of different phenomena (Austin, 1962; Gallagher, 2008; Gibson, 1979; Noë, 2005; Strawson, 1988; Zahavi, 2011). On a first interpretation, direct means non-representational. But believing that we perceive emotions does not imply accepting that perception is non-representational. Direct emotion perception and antirepresentationalism are conceptually independent claims, even if defendants of direct emotion perception are often also anti-representationalists (Hutto, 2008). However, direct perception claims are compatible with representationalism (Spaulding, 2011). A second interpretation equates direct with devoid of inference. Even so, characterizing direct perception as perception without inference only shifts the burden of clarification to the notion of inference. There are many ways to understand the notion of inference in the present debate. Here is a non exhaustive list of notions of inference: a) rational inferences involved in belief formation, b) cognitive non-rational inferences (for example associations), c) heuristics, d) perceptual expectancies (for example, amodal completion when seeing occluded figures), e) computational inferences. Which kind of inference is absent from direct perception? On a certain interpretation of "inference", the direct perception claim is a claim about the mechanisms of perception in general. This is the sense of direct at stake in the debate between Fodor and Pylsyhyn on the one hand, and Gibson on the other hand (Fodor \& Pylyshyn, 1981). In this sense indirect perception means that perception is computational, while direct perception means that perception is not mediated by computations. However this notion of direct is orthogonal to the notion of direct in direct emotion perception. Researchers from different approaches could agree that emotions can be the targets of perception, even if they disagree about the mechanisms involved. We need therefore an interpretation of direct that applies specifically to the case of emotion perception. In the particular case of direct emotion perception, direct means that it is devoid of extra-perceptual mechanisms (this is for example endorsed by Overgaard and Krueger, forthcoming). But in this case "direct perception" is just redundant with "perception" without a qualifier and it becomes identical to, or at least very similar to, the claim that one can simply see emotions. Therefore, I will use the two criteria of simple seeing as guiding principles: section 3 deals with the absence of extra-perceptual mechanisms (negative criterion), and section 4 deals with the subject's ability to differentiate emotional displays from the background (positive criterion). There are several advantages of an interpretation of direct perception as simple seeing. First, it is neutral in respect to the kind of cognitive mechanisms or inferences absent from perception, but it only says that extra-perceptual mechanisms are not necessary for simply seeing. Second, it allows contextual influence on perception, as long as there are no beliefs or concepts necessarily and constitutively involved. Third, it is largely independent from the notion of perception used in vision science, and is compatible with different approaches to the actual visual processes. A cautionary 
note before continuing: I will be switching back and forth between "not having beliefs" and "not having concepts" and I won't engage with the literature on what beliefs and concepts are. I will only assume the following. Beliefs (including perceptual beliefs) are propositional attitudes; states of simple seeing are non-propositional attitudes. Concepts are constituents of propositions. My aim is to provide some evidence that having beliefs about emotions and possessing the concepts for emotions are not necessary for simply seeing emotions.

\section{Is emotion perception free from extra-perceptual mechanisms?}

In this section, I will focus on the negative criterion for simply seeing something: the absence of extra-perceptual mechanisms (beliefs and concepts). In discussing direct emotion perception the notion of "contextual influence" (influence stemming from the immediate surrounding of the emotional facial display) has been used as the kind of mechanism that should be absent from emotion perception (this is suggested by Jacob, 2011). However, the notion of "context" is ambiguous. Contextual influence could range from folk-psychological beliefs about emotional situations, to beliefs about the setting and the objects present in the scene, to just contextual perceptual influence (such as lighting conditions on object perception). Unspecified contextual influence does not immediately undermine the claim that we perceive emotions. Whether or not contextual influence undermines the claim that we perceive emotions depends on how this contextual influence is understood. There are at least three options:

[Case 1] Beliefs about the display and its surroundings are necessary for having an experience of seeing emotions. There are no perceptual states with emotional content, only perceptual states with low-level features (the colors, shapes, orientations...of the display). Without the belief, there is no experience of seeing emotions. The impression we have of seeing emotions is not truly an experience of seeing emotions, but it is an experience as if we were seeing emotions.

[Case 2] Beliefs can influence perceptual states indirectly through attention. My belief that my friend has failed her exam influences my attentional mechanisms that make me fixate on certain traits of her face more than others. Perception together with attention constitutes the experience of perceiving emotions.

[Case 3] Beliefs can influence perceptual states with emotional content directly. One can have the perceptual state with emotional content and a belief about the context and this belief can change the perceptual state with emotional content. The strongest possibility is option 1, i.e. that without a cognitive mechanism one could not have an experience of the emotion at all: in this case, having a cognitive mechanism is a necessary condition for experiencing an emotion. Option 2 is weaker 
insofar as the cognitive mechanisms influence attention, and then attention to certain features gives rise to a perceptual experience of an emotion. In this case, the cognitive influence is merely indirect and one could still have the perceptual experience of an emotion without the accompanying belief, when attention is directed to the right features. An even weaker case is option 3, i.e. the claim that cognitive mechanisms can modulate an independently existing perceptual experience: for example, I see an angry face, but I am told that the person is thinking about her upcoming exam. Given this knowledge, I start to see the face no longer as angry but as fearful. Both my cognitive state (belief) and my perceptual state change. Or I see an angry face and then I notice a hiding gesture and a snake next to the person, so I start to see the face as fearful. Options 2 and 3 are both compatible with the alternative that the cognitive mechanism influences an already existing perceptual experience of an emotional face.

In sum, contextual influence can be used against direct perception of emotions when a) it is a case of extra-perceptual contextual influence and b) it is the strongest case of extra- perceptual contextual influence. Contextual influence has been used as a challenge to direct perception of emotions (Jacob, 2011). The challenge can be reconstructed as the following argument:

1. If there is contextual influence on emotion recognition, then one cannot directly perceive emotions.

2. There is contextual influence on emotion recognition (empirical evidence provided by (Aviezer et al., 2008)).

3. Therefore, one cannot directly perceive emotions.

To answer this challenge I will argue that the first premise is ambiguous between two notions of context: the strongest notion (case 1) and the two weakest notions (cases 2 and 3). A closer look at the empirical evidence shows that it supports only the weakest kind of contextual influence: withinperceptual contextual influence. In their 2008 experiment, Aviezer et al. showed subjects four vignettes, which included the same head with a disgusted expression on the face, but attached to different bodies that expressed either congruent or incongruent emotions (disgust, anger, sadness, or fear). They asked the subjects to pick the correct emotion to describe the expression from a list of emotions. According to the authors, the subjects based their choice on the bodily posture and the context (which included objects and scenes, such as a disgusting object or an object of sadness) and not on the expression of the face. The subjects were apparently not aware of the incongruence between the face and the body. The same results were obtained when the disgusted face was attached to a body expressing a positive emotion (pride). In the opinion of the authors of the study, 
this shows that context influences the recognition of the facial expression not only when the emotions shown are of the same valence, but also when emotions of opposite valence are shown. This interpretation is, of course, open to criticism. A reservation one might have with respect to this result is that since pride is not a basic emotion, it does not have a canonical facial expression. It may therefore be a special case.

But even if Aviezer et al.'s 2008 results do not apply to a switch in valence, they still hold for emotion recognition in the same valence category. Their third experiment tested the question of whether the influence of context was a perceptual effect or concerned post-perceptual processing, an issue that is obviously of much relevance to our present concerns. To test this, they used an eyetracking method, to see how subjects scanned the faces. It is known that when participants view angry faces they make more fixations on the eye region, and when they see disgusted faces they fixate on the eye and the mouth regions equally (Wong, Cronin- Golomb, \& Neargarder, 2005). Aviezer et al.'s eye-tracking experiment showed that when participants looked at the disgusted face on a disgusted body they fixated on the eye and the mouth regions equally (as was expected), but when the disgusted face was put on a body expressing anger they fixated the eye region more, as if they were looking at an angry face.

In 2012, the same researchers further tested the hypothesis that context can influence the perception of a face, and can switch the perceived valence of a facial expression from positive to negative (and vice versa) (Aviezer, Trope, \& Todorov, 2012). They showed participants a series of vignettes portraying tennis players in which the face of a loser was put on the body of a winner (both expressions were expressions of high arousal, i.e. of very intense emotion). The subjects were not aware of the switch. Bodily information again outweighed facial information. Subjects perceived the loser's face as happy instead of sad, when the face was put on a winner's body. To test once again whether or not the effect is perceptual, they asked participants to imitate the facial expression in the incongruent displays. Their motor simulation of the faces shifted as a function of the body's affective valence: they made a happy face when they saw the sad face of a loser on the body of a winner, and made a sad face when a winner's face was put on a loser's body. A set of new subjects had to judge the emotion expressed by the subjects imitating the facial expression of the people portrayed in the vignettes and they judged the subjects to imitate the incongruent emotion expressed by the body.

Aviezer et al.'s experiments show that the philosophers who tie the perception of facial expressions closely to the direct perception of emotion are operating with too simple a picture of how the process of perceiving emotions in others actually takes place. The role of context suggests there 
might be something wrong with the basic argument for our simply seeing emotions in virtue of seeing their facial manifestations. However, Aviezer et al.'s results do not show that concepts and beliefs influence perception of emotions. Given that premiss 2 is construed as an empirically testable prediction, we need other empirical evidence to support it. And so far such evidence is lacking.

The reason for this is that their interpretation of the contextual influence is problematic. The notion of context in their experiments includes not only bodily cues, but also various non-bodily objects present in the scene. It is far from clear that these two contextual cues, bodily postures and objects, are on the same level in the visual processing of emotional expressions. We might not want to extend the role of the body to the objects present in the scene.

Empirical research supports the central role of the body in perception. FMRI studies show distinct brain regions involved in the perception of faces and in the perception of faceless bodies, and neural populations are highly selective for bodies and unresponsive to faces (Peelen \& Downing, 2007). Patients with amygdala damage are impaired in recognizing anger in vignettes representing situations where a face is present (Adolphs, Tranel, \& Damasio, 2003). These patients however are as good as normal subjects at recognizing anger in vignettes presenting the same situation where the face has been digitally erased. While patients with amygdala damage are impaired at recognizing fear in isolated face presentation and in complex displays in which a face is present, they are surprisingly good at recognizing fear from bodily cues. Again, this indicates that in everyday interactions, facial information is only part of a more general perceptual setting from which emotion recognition stems. If context plays such an important role in recognizing emotions in everyday situations, and since we rarely see faces in isolation, it seems that emotion recognition is not limited to to seeing emotions in the face alone, but includes the body as well.

A possible objection is that these experiments study emotion recognition and not emotion perception properly speaking. However, the issue at stake is whether these experiments support the existence of necessary extra-perceptual influence on emotion perception previous to emotion recognition. My take is that they do not: they are compatible with the option that we simply see an emotion in the display and we form a belief on the basis of simple seeing. Even in the absence of beliefs about the settings, a subject could see the emotion in the face and body. However, since these experiments are conducted on neurotypical subjects with conceptual abilities, subjects are also immediately compelled to conceptualize that they see and to form beliefs about the display. The relevant question is whether this empirical evidence refutes that emotions can be possible targets of perception: the data do not support this claim. 
To sum up, Aviezer et al.'s experiments can be explained by appealing to the role of the body in perceiving emotions. Since they did not distinguish between bodily-context and inanimate- context, the effect in Aviezer et al.'s experiment might be an instance of extra-perceptual cognitive influence (for example a mechanism that links the presence of a trophy with joy, or the presence of a dirty diaper to disgust) or an instance of within-perception effects. Only the first option presents a real challenge to the claim that we perceive emotions rather than infer them. This is an empirical question and experimenters should find a way to present the same face and posture when only the objects in the scene vary, to test the influence of objects on perception of emotions. For the moment one can safely say that the body directly influences perception of emotional expressions, but not beliefs about the objects of emotions.

\section{Seeing emotions}

The positive criterion in Dretske's characterization of simple seeing tells us that the subject is able to differentiate the object from its immediate environment. This characterization is not of much help in providing strong criteria in favor of emotion perception. Instead I will apply the methods from the debate on the liberal content of perception to argue in favor of perception of emotions. Once a property is a potential candidate for liberal perceptual content, we need to use some criteria for deciding whether this property can be a target of perception or not. In this section, I will focus on some criteria that have been proposed as indicating perceptual content: a) distinctive phenomenal character, b) encapsulated processes, c) non-conceptual content, d) perceptual adaptation and afterimage effects, e) dissociation studies; f) neural substrata. These criteria represent presumptions in favor of perceptual content: states with contents that satisfy these criteria are perceptual. My aim is neither to establish that all emotions are perceived, nor it is to demonstrate that emotions are perceived as mental states, but it is to show that we can simply see some emotions.

My case in favor of emotion perception takes three steps. The first step is to show that we have at least one case of liberal perception: we perceive objects. Starting from the model of object perception, the second step is to show that we perceive faces and bodies. These two steps' function is to convince the reader that some high-level properties can be simply seen. The third step is to show that we also simply see emotions in faces and bodies (just as we simply see objects, faces and bodies) by applying the criteria outlined above.

\subsection{Seeing objects}

Usually no philosopher includes "object" as a perceptual property. Objects are not obviously lowlevel visual entities. It is true that the positive condition of simple seeing is the ability to 
differentiate $a$ from its surroundings, but this ability does not necessarily deliver us visual objects. In some cases, we have the experience of two objects, but there is no obvious differentiation between a foreground and a background (think of a sheet with a line in the middle and each side differently colored).

It is useful to distinguish between different notions of “object” (O'Callaghan, 2008): ordinary objects, formal objects, material objects, metaphysical objects, and visual objects. The only notion of object that is of interest here is the notion of visual (or perceptual) object, e.g. the object detected and tracked by the visual (perceptual) system. Vision does not track ordinary objects, because it identifies as objects things that would not be classified as ordinary objects (for example, a duck glued to a car) and it does not identify as objects some ordinary objects, for example keychains, because they are composed of several elements moving independently. Vision does not only track material objects either, since for the visual system non material objects such as holes, shadows, and rainbows, are also objects (O’Callaghan, 2008, p. 816).

Several pieces of experimental evidence suggest that vision parses the world into visual objects. Elementary properties such as colors and shapes are not presented as "free-floating" in experience, but as "bound" (Treisman, 1980, 1996). It has been argued that the things to which features are bound are not only locations, but also objects (sometimes called "proto- objects") (Cohen, 2004). Attention is not only directed at locations, but it also "sticks" to objects, as bounded, coherentlymoving entities (Pylyshyn, 2007). This was especially shown by the "reviewing paradigm", in which recognizing letters in boxes is facilitated when they re-appear bounded to the same object after a spatio-temporal change (Kahneman, Treisman, \& Gibbs, 1992).

These findings on adults' mid-level perception fit with the notion of "Spelke object" proposed by developmental psychologists to account for infants' object perception (Spelke, 1994). Indeed, Leslie and Scholl have suggested that adults' object-file system and infants' Spelke-objects do refer to the same natural kind (Leslie \& Scholl, 1999).

Even if Spelke herself claims that her data show that infants possess the innate concept of object, these data better fit with the idea that objects are seen. First, they are sensitive to visual constraints: only bounded, coherently moving wholes are tracked as objects. Visual tracking is easily disrupted (as opposed to conceptual tracking). Second, they initiate spontaneous action (like reaching). Third, they have non-conceptual content. One of the arguments used by Spelke in favor of the conceptualist view is that the tracking of Spelke- objects survives in the absence of retinal stimulation, when the object disappears behind a barrier. This means that object representations are amodal (independent from a given modality, visual in this case). However, surviving through 
occlusion does not imply that these representations are conceptual, even if it means they are not sensory. The interpretation of the phenomenon depends on one's theory of amodal completion. Briscoe distinguishes between non-cognitive amodal completion and cognitive amodal completion: First, the informational basis for the perception of a contour or surface is not limited to its optical projection in the retinal image. Texture deletion under perspective transformation, binocular disparity, and T-junctions are all sources of ecologically valid visual information for the existence of occluded object-features. The absence of sensory stimulation from a feature $F$ thus does not always mean the absence of visual information for $\mathbf{F m}$ [emphasis added]. Second, there is empirical evidence that sources of visual information for occlusion are actually used by NAcompletion [non-cognitive amodal completion] mechanisms to form representations of occluded object-regions in early visual processing areas such as primary visual cortex. Last, the representational outputs of NA-completion mechanisms play a fundamental role in the perceptual grouping of visible surface regions in depth. In particular, they help causally to explain our visual experience of partially occluded objects as coherent, unified wholes. (Briscoe, 2011, p. 161)

To sum up, objects can be part of perceptual content even if this does not mean that they are seen as objects. The distinction between visual objects and the folk-psychological concept of object is crucial.

\subsection{Seeing faces (and bodies)}

Objects are not the only high-level properties that can be a target of perception. Also faces and bodies are. For reasons of space, I will focus only on faces and assume that the discussion of faces can be extended to bodies as well (see section 3 on evidence in favor of body perception).

Researchers have primarily pursued the search for the perceptual module underlying face perception (Kanwisher \& Yovel, 2006). Evidence of a face module comes from: developmental studies (Goren, Sarty, \& Wu, 1975; Johnson, Dziurawiec, Ellis, \& Morton, 1991); brain imaging studies (Kanwisher, 2000); prosopagnosia (Farah, 2004); evolutionary psychology (McKone, Crookes, Jeffery, \& Dilks, 2012); and perceptual effects specific to face perception, such as the 'inversion effect' (Valentine, 1988).

Kanwisher and colleagues study an area of the brain, called the FFA (fusiform face area), located in the posterior right hemisphere. This area is particularly active when subjects look at face stimuli, but shows low activation when subjects view other stimuli, including living beings, hands, and houses (Grill-Spector, Knouf, \& Kanwisher, 2004; Kanwisher, 2000; Kanwisher \& Yovel, 2006). 
The face module hypothesis has been criticized. The main alternative hypothesis for the role of the FFA is that the area is dedicated to the processing of objects of expertise, not only of faces (Diamond \& Carey, 1986; Gauthier, Behrmann, \& Tarr, 2004; Gauthier \& Tarr, 1997). Gauthier and Tarr $(2004 ; 1997)$ argue that it is possible to see both the inversion effect and the activation of the FFA after training individuals to recognize a set of new abstract stimuli, called 'Greebles'. One methodological issue with the Greebles experiment is that the stimuli, albeit abstract, share some similarities with faces. Humans are very much subject to pareidolia, the tendency to see faces everywhere. A way to resolve the controversy between the two hypotheses (that FFA is the face area and that FFA is the expertise area) is to offer a sophisticated definition of the notion of special processing. Young distinguishes between two interpretations of the notion of special: on the stronger interpretation 'special' means unique and it implies that only faces elicit the activation of the FFA. On the weaker interpretation, having a dedicated system does not mean it cannot be used in other domains (Young, 1998, p. 68). He concludes that "[faces] are special but [...] they are not unique" (Young, 1998, p. 89).

Tsao and Freiwald (2006) study the monkey analog of the FFA using both brain imaging and single cell recordings: ninety-seven percent of neurons in this region were selectively responsive to faces over non-face stimuli thus confirming at least the weak version of special processing for faces. Behavioral evidence such as that discussed above, in addition to activation of the FFA, points to an innate preference for faces. Newborns prefer to follow a human-like face compared to a blank or a scrambled face (Johnson et al., 1991). This preference is also present in newly hatched chicks (Rosa- Salva, Regolin, \& Vallortigara, 2010). Faces (and bodies) are thus high-level properties that are represented in perception. But do we also perceive the emotions they express?

\subsection{Seeing emotions}

The final step is to show that emotions in faces and bodies can be perceived. Basing on the distinction between seeing objects and conceptualizing objects, I distinguish between visual emotions and folk-psychological emotions (emotions conceptualized as mental states). The aim of this section is to show that emotion perception respects six criteria of perceptuality introduced at the beginning of section 4 .

Concerning phenomenology, for example, we cannot help having the experience of an emotion even when we know that this experience is an illusion. When we watch a play we have the experience of seeing sadness in the actor's expression even if we know that she is only pretending.

I have implicitly argued for encapsulation in emotion perception is section 3: for the time being we 
lack clear-cut evidence of the necessity of non within-perceptual influence for emotion perception. In addition, emotion perception is a very fast, automatic, involuntarily process that has characteristic breakdowns (Fodor, 1983), as we will see from the discussion of the empirical literature.

The non-conceptual format is often said to characterize perceptual states (Crane 1988). Prima facie evidence comes from the fact that animals and pre-verbal infants seem to detect and imitate emotions (Meltzoff \& Moore, 1977), even if they supposedly do not possess concepts. Each emotion is accompanied by a specific way we attend to the face; and in addition bodily contextual influence can change the attentional pattern (Aviezer et al. 2012). According to Kelly (2001), nonconceptual perceptual content shows a context-dependency no concept can account for. He identifies two examples of context-dependencies. First, we can have different experiences of the same color under different illumination conditions, despite the fact that it is the same color in the world (color constancy). This shows for him that the content of a color experience is not complete without including lighting conditions (the context), but normally concepts do not refer to the context. Second, we can wonder whether the blue of the carpet is the same blue as the blue of the steel (object dependency). While our perceptual experiences can appear to be different for the carpet blue and for the steel blue (even though they reflect exactly the same wavelengths), the concept "blue" cannot distinguish between these two experiences (Kelly, 2001). We can draw a parallel between the role of the light for the perception of the same color under different lighting conditions and the role of body for the perception of emotions: when the contextual (bodily) conditions change, the non- conceptual content that represents the emotion also changes. ${ }^{1}$

Additional support comes from three empirical lines of evidence: a) perceptual effects; b) dissociations and lesion studies; c) neural substrata.

Both perceptual adaptation and after-image effects occur in the case of emotions. Adaptation to a face biases the perception of the expression away from the adapting expression (Butler, Oruc, Fox, \& Barton, 2008). For example, after looking at a sad face, the neutral face looks happy (Javadi \& Wee, 2012; Pell \& Richards, 2011; Webster \& MacLeod, 2011). Crucially, the subjects do not simply adapt to low-level properties, such as shapes and colors, but they adapt to high-level

1 One could argue that concepts also exhibit some context-dependency. For example in Rozin et al.'s (1990) experiments, subjects were more reluctant to drink water from a bottle with the label "poison" even if they knew for sure that the bottle contained water. Context-independency might come in degrees, with non-conceptual content showing the lowest rate of context-independency and conceptual content showing higher rates of context-independency. Prototypes (Rosch, 1983) might be intermediate between non-conceptual and full-blown conceptual content. 
properties of the display (Rutherford, Chattha, \& Krysko, 2008). Perceptual adaptation is considered a typically perceptual effect (Block, 2014).

A skeptic about this evidence could say that it shows that we perceive merely facial and bodily expressions (behaviors) and not emotions. However, imagine a similar objection in the case of object perception: all the evidence on Spelke-objects shows that we see traits of objecthood and not objects. This seems contra-intuitive: we visually track objects, even if we do not track them as falling under the concept "object". In addition, it has been argued that we perceive natural kinds even if this does not imply that we perceive the underlying DNA structure that makes the perceived token of a certain natural kinds a member of the natural category (Siegel, 2013). We can perceive pine trees even if we do not perceive pinetreehood. Similarly we can perceive emotions even if we do not perceive them as mental states, i.e. without recognizing them as mental states or perceiving them as mental states. For instance it is usually assumed that an emotional mental state is intrinsically relational: one is afraid of the dog. But perception does not have to take into account the relational aspect to perceive emotions, just as we can perceive the moon without knowing wether it is made of cheese or rock. This way we can support sub-thesis 2 without supporting (yet) sub-thesis 1 (see Section 1).

Empirical evidence seems to point toward the idea that facial and bodily expressions elicit automatic reactions that are congruent with the emotion seen (retreat for fearful emotions and advancing for joyful emotions). In recent experiments, Borgomaneri and colleagues (2014) used TMS (transcranial magnetic stimulation) to explore motor excitability during observation of emotional scenes before the onset of late motor modulation (at $300 \mathrm{~ms}$ ). They found that between 100 and $125 \mathrm{~ms}$ there is already a suppression of the inappropriate motor reaction to the emotion present in the display, and at around $150 \mathrm{~ms}$ an orienting response (action preparation).

Can we study emotion perception separately from emotion categorization and recognition? One possibility is to look at evidence on dissociations and lesions studies. However, this evidence is not easy to interpret. Several studies link the amygdala with processing and recognition of negative emotions (Davis, 1992). Damage to the amygdala results in an impaired recognition of negative emotions when they are expressed in the face (Adolphs, Tranel, Damasio, \& Damasio, 1994): patients with this damage are not able to attribute fear to fearful faces, even if they perfectly master the concept of fear, and can correctly apply it to verbally presented situations of fear. Does this have implications for emotion perception? They do not spontaneously attend to the eye region (the area average subjects attend to when looking at a fearful face), which seems to point toward an additional disruption in perceptual processing. The question now is whether in these patients both 
perception and recognition are disrupted, or only recognition is disrupted. Tsuchiya and colleagues (2009) found that a patient with complete bilateral amygdala lesions (unable to recognize and classify visual stimuli of faces showing fear) was nonetheless able to detect the same fearful faces in rapid presentations below the threshold of consciousness. The subject was presented with two pictures: a fearful face and a non-fearful face, for $40 \mathrm{~ms}$, and had to press a key to identify which was the fearful face. While unable to recognize the fearful face with unlimited time to decide, in the forced choice condition, the patient with amygdala damage correctly selected the fearful face. What is the role of amygdala if it is not to underlie perception of emotions? Is its role to mediate between subliminal perception and conscious perception? Or is its role to mediate between perception and the ability to consciously categorize the emotional displays? Or is it to simulate another's fear? The likely interpretation is then that they can perceive emotions, and maybe experience them, in the absence of higher order access. But in this case it is not possible to use evidence from amygdala damage to argue that perception of emotion is disrupted. Rather, a more accurate interpretation of the disruption appears to be, at the very least, that it is the cognitive processing that does the later, post- perceptual explicit recognition and attribution of the stimulus that is impaired.

In many cases, claims in favor of emotion perception are based on the presence of resonance mechanisms in seeing emotional displays, such as those supposedly supported by the amygdala (Gallagher, 2007). But the appeal to resonance mechanisms leads to unfortunate consequences as Jacob (2011) points out: "As I see it, the problem is that the direct- perception model of social perception faces a dilemma: if what underlies social perception is MN [motor neuron] activity, then social perception cannot be both resonant and enactive." (Jacob, 2011, p. 529). The dilemma stems from the opposition between an enactive mode, in which we are actively engaged as actors in the social world, and a resonant mode in which the recipient of the perceptual stimuli is a passive observer who duplicates the state of the other.

In order to choose one side, we should rethink the neural substrata of emotion perception in light of empirical data. Empirical studies locate emotion perception in the STS (superior temporal sulcus) (Said, Moore, Engell, Todorov, \& Haxby, 2010). Note that the STS' activation follows the activation of primary visual areas, but it is still considered as part of the visual processing of the stimuli. The STS sends reciprocal projections to the inferior parietal cortex, which is in turn connected to F5 (mirror area in monkeys). However, the STS does not possess mirror properties and there is no direct connection between area F5 and the STS (Keysers \& Perrett, 2004).

Evidence from Borgomaneri and colleagues answers to Jacob's dilemma: it is plausible that social (emotion) perception is independent from mirroring activity. The researchers suggest that motor 
reaction and orienting are present earlier than motor resonance (which in their opinion accounts for empathy). Therefore, these data (weakly) support the enactive mode of emotion perception. The upshot, however, is that direct perception has to abandon the appeal to resonance mechanisms in support of emotion perception.

But does this evidence support early understanding, categorization and attribution of emotions? It depends on the notions of understanding, categorization and attribution one wants to endorse, but I would like to sketch some motivations for a negative answer. Early motor responses might be instances of reflexes and primitive reactions that fall short of recognition and attribution.

\section{Perceiving emotions without mindreading them}

Direct perception of emotions is often introduced as an alternative (or additional) mechanism for mindreading (Zahavi, 2011). However, the discussion of sections 2 and 3 leads to some skepticism about this claim. Mindreading is usually defined as "the attribution of a mental state to self or other. [...] [T] o mindread is to form a judgment, belief, or representation that a designed person occupies or undergoes [...] a specific mental state or experience. [...] [T] o attribute a mental state, the judgement must deploy a mental concept or category [emphasis added]" (Goldman, 2009, p. 312).

Remember the definition of simple seeing: to simply see a subject does not need beliefs (judgements) or concepts. The evidence introduced in sections 3 and 4 is thus entirely compatible with seeing emotions without seeing them as mental states, i.e. without being able to categorize or conceptualize them under mental concepts or categories. Recall the contrast between visual objects (Spelke-objects) and the ordinary concept of an object. Anything that satisfies specific perceptual constraints (cohesive, bounded, persistent and coherently moving whole) is picked up by the visual system as a Spelke-object (Spelke, 1990), even if it does not fall under our folk-psychological concept of "ordinary object".

Cognition works on the defeasible assumption that Spelke-objects are ordinary objects, but this assumption might be revised in light of new evidence, for example when we learn that we have been tracking two distinct objects (a duck and a truck) all along. Similarly, I suggest to distinguish between visual emotions (emotions that are part of perceptual experience) and emotions as folkpsychological concepts. Possessing the folk psychological concept of an emotion can account for our understanding of many of the properties commonly attributed to emotions and not captured by perception, e.g. that emotions have intentional objects, that actors can pretend to have them, that emotions can be motivating, etc. But it does not follow from this that emotions are not part of perceptual experience and that only low level, local, features are perceived when we see a happy 
person. That follows is that in some cases we can see happiness in a person's face and body, without seeing the person as happy.

Of course, this does not rule out the possibility of immediate perception-based understanding and attribution. However it demonstrates that evidence in favor of emotion perception is not evidence in favor of emotion perception as a mechanism of emotion understanding and attribution. If we accept that to mindread one needs a mental concept or category in order to form a belief or judgement, then it is not sufficient to show that we can perceive $\mathrm{x}$ to demonstrate that it is thanks to perception that we recognize $\mathrm{x}$ as an $\mathrm{x}$. Other mechanisms are still needed to form judgements based on perceptual experiences of emotions.

Thus, the question becomes: how are social perceptions and social judgements connected? Showing that emotions can be perceived is only a first step in providing an alternative account of mindreading.

\section{Conclusion}

Let us take stock on the discussion so far. In the introduction I presented the debate between two options: emotions are perceived vs. emotions are inferred via non-perceptual mechanisms. I appealed to theoretical and empirical considerations to show that emotions can simply be seen. I also argued against the claim that perceiving emotions can give us an alternative mechanism for mindreading emotions. I suggested that emotions could be part of perceptual experience, even if we do not immediately recognize, classify, or attribute them to others as mental states. The account presented in this paper conciliates some aspects of both options for emotion recognition: it concedes to direct perception that emotions are perceived, but it does not endorse the idea that perceiving emotions accounts for mindreading them.

\section{References}

Adolphs, R., Tranel, D., \& Damasio, A. R. (2003). Dissociable neural systems for recognizing emotions. Brain and Cognition, 52(1), 61-69.

Adolphs, R., Tranel, D., Damasio, H., \& Damasio, A. (1994). Impaired recognition of emotion in facial expressions following bilateral damage to the human amygdala. Nature, 372(6507), 669-672.

Austin, J. L. (1962). Sense and Sensibilia. Oxford University Press.

Aviezer, H., Hassin, R. R., Ryan, J., Grady, C., Susskind, J., Anderson, A., ... Bentin, S. (2008). Angry, Disgusted, or Afraid?: Studies on the Malleability of Emotion Perception. Psychological Science, 19(7), 724-732. doi:10.1111/j.1467-9280.2008.02148.x 
Aviezer, H., Trope, Y., \& Todorov, A. (2012). Holistic person processing: faces with bodies tell the whole story. Journal of Personality and Social Psychology, 103(1), 20.

Block, N. (2014). Seeing- As in the Light of Vision Science. Philosophy and Phenomenological Research, 89(1).

Borgomaneri, S., Gazzola, V., \& Avenanti, A. (2014). Transcranial magnetic stimulation reveals two functionally distinct stages of motor cortex involvement during perception of emotional body language. Brain Structure and Function, 1-17.

Briscoe, R. (2011). Mental imagery and the varieties of amodal perception. Pacific Philosophical Quarterly, 92(2), 153-173.

Butler, A., Oruc, I., Fox, C. J., \& Barton, J. J. (2008). Factors contributing to the adaptation aftereffects of facial expression. Brain Research, 1191, 116-126.

Cohen, J. (2004). Objects, places, and perception. Philosophical Psychology, 17(4), 471-495.

Davis, M. (1992). The role of the amygdala in fear and anxiety. Annual Review of Neuroscience, 15(1), 353-375.

Diamond, R., \& Carey, S. (1986). Why faces are and are not special: an effect of expertise. Journal of Experimental Psychology: General, 115(2), 107.

Dretske, F. (1969). Seeing And Knowing (Vol. 21). Chicago: University Of Chicago Press.

Dretske, F. (1973). Perception and other minds. Nô̂s, 7(March), 34-44.

Farah, M. J. (2004). Visual agnosia. MIT press.

Fodor, J. A. (1983). The Modularity of Mind. MIT Press. Fodor, J. A., \& Pylyshyn, Z. W. (1981). How direct is visual perception? Some reflections on Gibson's “ecological approach." Cognition, 9(2), 139-96.

Gallagher, S. (2007). Simulation trouble. Social Neuroscience, 2(3-4), 353-365.

Gallagher, S. (2008). Direct perception in the intersubjective context. Consciousness and Cognition, $17(2), 535-543$.

Gallagher, S. (2014). In your face: Trascendence in embodied interaction. Frontiers in Human Neuroscience, $8,495$.

Gauthier, I., Behrmann, M., \& Tarr, M. J. (2004). Are Greebles like faces? Using the neuropsychological exception to test the rule. Neuropsychologia, 42(14), 1961-1970. 
Gauthier, I., \& Tarr, M. J. (1997). Becoming a "Greeble" expert: Exploring mechanisms for face recognition. Vision Research, 37(12), 1673-1682.

Gibson, J. J. (1979). The Ecological Approach to Visual Perception. Houghton Mifflin.

Goldman, A. (2006). Simulating Minds: The Philosophy, Psychology, and Neuroscience of Mindreading (Vol. 144). Oxford University Press.

Goldman, A. I. (2009). Mirroring, simulating and mindreading. Mind and Language, 24(2), 235252.

Goren, C. C., Sarty, M., \& Wu, P. Y. (1975). Visual following and pattern discrimination of facelike stimuli by newborn infants. Pediatrics, 56(4), 544-549.

Grill-Spector, K., Knouf, N., \& Kanwisher, N. (2004). The fusiform face area subserves face perception, not generic within-category identification. Nature Neuroscience, 7(5), 555-562.

Hawley, K., \& Macpherson, F. (2011). The Admissible Contents of Experience. Wiley- Blackwell.

Hutto, D. D. (2008). Articulating and understanding the phenomenological manifesto. Abstracta Linguagem, Mente E Ação, 10(19), 10-19.

Jacob, P. (2011). The Direct-Perception Model of Empathy: a Critique. Review of Philosophy and Psychology, 2(3), 519-540.

Jacob, P., \& Jeannerod, M. (2003). Ways of Seeing: The scope and limits of visual cognition. OUP Oxford.

Javadi, A. H., \& Wee, N. (2012). Cross-category adaptation: objects produce gender adaptation in the perception of faces. PloS One, 7(9), e46079.

Johnson, M. H., Dziurawiec, S., Ellis, H., \& Morton, J. (1991). Newborns’ preferential tracking of face-like stimuli and its subsequent decline. Cognition, 40(1), 1-19.

Kahneman, D., Treisman, A., \& Gibbs, B. J. (1992). The reviewing of object files: Object- specific integration of information. Cognitive Psychology, 24(2), 175-219.

Kanwisher, N. (2000). Domain specificity in face perception. Nature Neuroscience, 3, 759- 763.

Kanwisher, N., \& Yovel, G. (2006). The fusiform face area: a cortical region specialized for the perception of faces. Philosophical Transactions of the Royal Society B: Biological Sciences, 361(1476), 2109-2128.

Kelly, S. D. (2001). The non-conceptual content of perceptual experience: Situation dependence 
and fineness of grain. Philosophy and Phenomenological Research, 62(3), 601- 608.

Keysers, C., \& Perrett, D. I. (2004). Demystifying social cognition: a Hebbian perspective. Trends in Cognitive Sciences, 8(11), 501-507.

Krueger, J. (forthcoming). Emotions and Other Minds. In R. Campe \& J. Weber (Eds.), Interiority/Exteriority: Rethinking Emotion. Walter de Gruyter.

Krueger, J., \& Overgaard, S. (forthcoming). Seeing subjectivity: defending a perceptual account of other minds. Protosociology.

Leslie, A. M., \& Scholl, B. J. (1999). Modularity, development and "theory of mind." Mind and Language, 14(1), 131-153.

Michotte, A. (1954). La perception de la causalité. Publications universitaires de Louvain.

McKone, E., Crookes, K., Jeffery, L., \& Dilks, D. D. (2012). A critical review of the development of face recognition: Experience is less important than previously believed. Cognitive Neuropsychology, 29(1-2), 174-212.

Meltzoff, A. N., \& Moore, M. K. (1977). Imitation of facial and manual gestures by human neonates. Science, 198(4312), 75-78.

Milner, A. D., \& Goodale, M. A. (1995). The Visual Brain in Action. Oxford University Press.

Noë, A. (2005). Action in Perception. The Mit Press.

O’Callaghan, C. (2008). Object Perception: Vision and Audition. Philosophy Compass, 3(4), 803829.

Peelen, M. V., \& Downing, P. E. (2007). The neural basis of visual body perception. Nature Reviews Neuroscience, 8(8), 636-648.

Pell, P. J., \& Richards, A. (2011). Cross-emotion facial expression aftereffects. Vision Research, 51(17), 1889-1896. Pylyshyn, Z. W. (2007). Things and Places: How the Mind Connects with the World. The Mit Press.

Rosa- Salva, O., Regolin, L., \& Vallortigara, G. (2010). Faces are special for newly hatched chicks: evidence for inborn domain- specific mechanisms underlying spontaneous preferences for face- like stimuli. Developmental Science, 13(4), 565-577.

Rosch, E. (1983). Prototype classification and logical classification: The two systems. New Trends in Conceptual Representation: Challenges to Piaget's Theory, 73-86. 
Rozin, P., Markwith, M., \& Ross, B. (1990). The sympathetic magical law of similarity, nominal realism and neglect of negatives in response to negative labels. Psychological Science, 1(6), 383384.

Rutherford, M. D., Chattha, H. M., \& Krysko, K. M. (2008). The use of aftereffects in the study of relationships among emotion categories. Journal of Experimental Psychology: Human Perception and Performance, 34(1), 27.

Said, C. P., Moore, C. D., Engell, A. D., Todorov, A., \& Haxby, J. V. (2010). Distributed representations of dynamic facial expressions in the superior temporal sulcus. Journal of Vision, 10(5), 11. Siegel, S. (2010). The Contents of Visual Experience. Oxford University Press.

Siegel, S. (2013). Replies to Campbell, Prinz, and Travis. Philosophical Studies, 163(3), 847-865.

Spaulding, S. (2011). A Critique of Embodied Simulation. Review of Philosophy and Psychology, 2(3), 579-599.

Spaulding, S. (in preparation). On Direct Social Perception.

Spelke, E. (1994). Initial knowledge: six suggestions. Cognition, 50(1-3), 431-445.

Strawson, P. F. (1988). Perception and its objects. In J. Dancy (Ed.), Perceptual Knowledge. Oxford University Press.

Treisman, A. (1980). A feature integration theory of attention. Cognitive Psychology, 12, 97- 136.

Treisman, A. (1996). The binding problem. Current Opinion in Neurobiology, 6, 171-8.

Tsao, D. Y., Freiwald, W. A., Tootell, R. B., \& Livingstone, M. S. (2006). A cortical region consisting entirely of face-selective cells. Science, 311(5761), 670-674.

Tsuchiya, N., Moradi, F., Felsen, C., Yamazaki, M., \& Adolphs, R. (2009). Intact rapid detection of fearful faces in the absence of the amygdala. Nature Neuroscience, 12(10), 1224.

Valentine, T. (1988). Upside- down faces: A review of the effect of inversion upon face recognition. British Journal of Psychology, 79(4), 471-491.

Webster, M. A., \& MacLeod, D. I. (2011). Visual adaptation and face perception. Philosophical Transactions of the Royal Society B: Biological Sciences, 366(1571), 1702-1725.

Wong, B., Cronin-Golomb, A., \& Neargarder, S. (2005). Patterns of visual scanning as predictors of emotion identification in normal aging. Neuropsychology, 19(6), 739.

Young, A. W. (1998). Face and mind. Oxford University Press Oxford. 
Zahavi, D. (2011). Empathy and Direct Social Perception: A Phenomenological Proposal. Review of Philosophy and Psychology, 2(3), 541-558. 